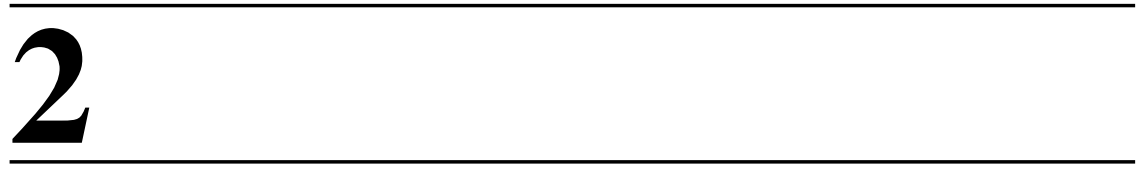

\title{
NANOSIZED DELIVERY SYSTEMS FOR TISSUE REGENERATION
}

\author{
Goksu Cinar, Didem Mumcuoglu, Ayse B. Tekinay, \\ AND Mustafa O. Guler \\ Institute of Materials Science and Nanotechnology, National Nanotechnology \\ Research Center (UNAM), Bilkent University, Ankara, Turkey
}

\subsection{INTRODUCTION}

Repair and regeneration of damaged tissue is an important clinical need since millions of people all over the world are suffering from tissue and organ failure (Rice et al., 2013; Wei and Ma, 2008). Although tissue regeneration processes and components are quite different depending on tissue properties, the fundamentals of regeneration process involves cells, cellular microenvironment, and biological signals. Hence, the strategies in tissue regeneration focus on cell-based therapies, regenerative biomaterials as scaffolds, and delivery of biological signals to regenerating site. The integration of these strategies and the components with natural tissue healing stages is important to achieve successful therapies in clinical applications (Howard et al., 2008).

Controlled delivery of biologics such as growth factors (GFs), cytokines, nucleic acids, or siRNA is the focus of growing interest in tissue regeneration applications since these biomacromolecules serve highly specific and complex functions in cellular processes compared to small-molecule

Therapeutic Nanomaterials, First Edition. Edited by Mustafa O. Guler and Ayse B. Tekinay.

(C) 2016 John Wiley \& Sons, Inc. Published 2016 by John Wiley \& Sons, Inc. 
synthetic drugs (McCall et al., 2011; Vermonden et al., 2012). Although therapeutic approaches in tissue regeneration focus on biologics, the clinical applications are highly limited due to the fragile nature and instabilities of the molecules. Delivery systems can increase therapeutic applicability of biologics sustaining a suitable environment for threedimensional conformations and protection from enzymatic degradation during transportation to the regenerating site. In addition, these systems aim to mimic natural release mechanisms, conditions, and therapeutic dosage of biologics, which are controlled by distinct patterns and enzymatic reactions in cellular microenvironment (Tessmar and Göpferich, 2007). Stimuli-response of the nanosized carriers is also an important property to decrease side effects and effective dosage of the molecules necessary for the regeneration in tissue-specific conditions (AlvarezLorenzo and Concheiro, 2014).

Advances in material science and integration with nanotechnology provide us a new generation of biodegradable and biocompatible nanosized delivery systems with control over size, shape, and multifunctionality (Panyam and Labhasetwar, 2003; Zhang et al., 2013). Nanosized delivery systems can be designed as lipid-based, inorganic, polymeric, or multifunctional hybrid systems including liposomes, polymeric micelles, mesoporous silica nanoparticles, nanogels, or nanocomplexes with sizes varying between 1 and $200 \mathrm{~nm}$ (Khandare et al., 2012). Nanosized delivery systems provide tissue penetrating ability, reduced toxicity, and enhanced permeation and retention (EPR) in regenerating tissues (Gu et al., 2011). These systems can be injected to the bloodstream and extend the release of biologics protecting them from proteolytic cleavage and chemical degradation. EPR effect also provides passive targeting for the nanosized carriers increasing circulation time in the bloodstream. Improved solubility of hydrophobic compounds can be obtained via nanosized delivery systems (Mishra et al., 2010). In addition, nanosized delivery systems can be integrated into three-dimensional scaffolds sustaining controlled release and biocompatible microenvironment for regenerating cells (Wei and Ma, 2008).

Multifunctional nanosized delivery systems for biologics have been developed using both natural and synthetic polymers. Synthetic polymers enable sustained release of biologics over a period of days to several weeks based on different release mechanisms including concentration, degradation, affinity or stimuli controlled, and also combinations of them. FDA-approved synthetic polymers such as poly(lactide-co-glycolide) (PLGA) have been used for developing nanosized delivery systems due to their biocompatibility and tunable physical and chemical properties (Golub et al., 2010). PLGA nanoparticles can be hydrolyzed into biodegradable 
metabolites: lactic and glycolic acid at acidic conditions (Kumari et al., 2010). In addition, there are examples of polymeric nanoparticles developed as biological delivery systems using poly(lactic acid) (PLA), poly(D,Lglycolide) (PLG), poly- $\varepsilon$-caprolactone (PCL), and poly(alkyl cyanoacrylates) for tissue regeneration (Kim et al., 2014). Although some of these synthetic nanosized polymeric systems reveal biodegradable properties, degradation products can be toxic compounds, and degradation process takes longer time periods. Moreover, formulation conditions and encapsulation steps may require organic solvents and high ionic strength, creating a harsh environment for biologics.

On the other hand, natural polymers can form biodegradable polymeric nanostructures for biomacromolecule delivery at mild conditions, and these nanostructures are functionalized with different delivery strategies for targeting and internalization. For these purposes, both protein-based natural polymers such as gelatin, collagen, albumin, and elastin and hydrophilic polysaccharides including alginate or chitosan have been used to obtain colloidal nanostructures. Compared to synthetic polymeric nanostructures, natural nanocarriers can be easily degraded by digestive enzymes and degradation products not harmful. However, the hydrophilic nature of these nanosized carriers leads to burst release of biologics when biologics are immobilized within the system via noncovalent interactions or short-term release profiles can be obtained due to easier biodegradability compared to synthetic polymeric nanosized delivery systems. In addition, contamination risk is higher since the sources of these compounds are natural organisms.

Molecular assemblies of both synthetic and natural compounds are intriguing nanosized delivery structures for biologics. Colloidal nanostructures such as liposomes, polymeric nanoparticles, block copolymer micelles, and dendrimers can be designed as stimuli responsive, and delivery mechanisms can be enhanced via internal and external factors including $\mathrm{pH}$, temperature, or redox microenvironment (Fleige et al., 2012; Ganta et al., 2008). Intravenous administration of these colloidal nanosized systems also makes them suitable carriers for tissue regeneration applications (Alyautdin et al., 2014). In addition, the stimulated effects of gold nanoparticles between 20 and $50 \mathrm{~nm}$ size on osteogenic differentiation of stem cells and osteoblast-like cells have been showed in different studies (Heo et al., 2014; Ko et al., 2015).

In this chapter, we focus on advanced delivery of biologics including GFs, cytokines, genes, or siRNAs using a variety of nanosized systems for different regeneration applications focusing on bone, cartilage, nervous system, and muscle regeneration strategies. The limitations of biologics delivery and alternative strategies for overcoming recent problems are underlined presenting recent examples from the literature. In addition, 
specific targeting and cellular internalization strategies of biologics delivery for tissue regeneration are discussed for providing future perspectives to the readers in this field.

\subsection{DELIVERY OF PROTEIN THERAPEUTICS WITH NANOCARRIERS FOR TISSUE REGENERATION}

\subsubsection{GFs and Cytokines}

As biological regulatory signals in variety of cellular responses, GFs are important components for controlling and directing tissue formation, maintenance, and regeneration. In addition, these biological signals direct crucial tissue regeneration processes such as angiogenesis and bone or granulation tissue formation (Eichmann and Simons, 2012; Laurencin et al., 2014; Ponte et al., 2007). Although the functions and importance of many GFs in tissue regeneration are well known, the clinical applications and therapeutic efficiency of these biologics are limited due to their short lifetime, production costs, and safety concerns for immunogenic responses (Martino et al., 2014; Rice et al., 2013). To increase their lifetime and facilitate controlled release of biologically active GFs over an extended time period for therapeutic applications, nanosized delivery strategies have been developed using different materials.

In living organisms, GFs are stabilized in extracellular matrix (ECM) via interactions of highly sulfated ECM components such as glycosaminoglycan (GAG) side chains. GFs can be immobilized on nanosized carriers by either noncovalent or covalent interactions (Chen et al., 2010). Polymeric nanoparticles with functional groups can be designed by sustaining these specific interactions for immobilization and affinity-controlled GF delivery (Wang and von Recum, 2011). Natural polyanionic polysaccharides such as heparin and chondroitin sulfate can form nanocomplexes with natural or synthetic polycationic polymers, and polyelectrolyte complex nanoparticles are used to deliver heparin-binding GFs including fibroblast growth factor (FGF) family and transforming growth factor- $\beta$ (TGF- $\beta$ ) superfamily (Place et al., 2014). Natural polymers including proteins and polysaccharides can be modified with several functional groups and form colloidal nanostructures, which are also suitable candidates for delivering GFs via noncovalent interactions or covalent crosslinking. Gelatin is a well-known natural protein-based polymer with excellent biocompatibility and controllable biodegradability. It can be modified as either negatively or positively charged and enable polyion complexation with several GFs (Young et al., 2005). 
Angiogenesis is a critical process in tissue restoration and constitutes of establishing a vascular system to supply required oxygen and nutrition to the regenerating site. Vascular endothelial growth factor (VEGF), which is a member of the cystine knot family, is an important biological factor for mediating angiogenesis in wound healing and myocardial ischemia treatments (Crafts et al., 2015). Different delivery strategies are developed for sustained release of bioactive VEGF to the regenerating site. In one study (Golub et al., 2010), VEGF-loaded PGLA nanoparticles enhanced blood vessel growth via sustained delivery for cardiovascular medicine applications. Polyelectrolyte nanosized complexes formed by coacervation of VEGF-bound dextran sulfate with different polycations such as chitosan, polyethylenimine, or poly-L-lysine were shown to have high encapsulation efficiency for the regeneration applications (Huang et al., 2007). In addition, it was shown that GAG-based polyelectrolyte nanocomplexes are quiet stable delivery systems at physiological conditions and show resistance to high ionic strength for in vivo applications (Novoa-Carballal et al., 2014). In another example, in vivo therapeutic revascularization was obtained via VEGF-loaded heparin-functionalized nanoparticle-fibrin complexes in a rabbit ischemic hind limb model (Chung et al., 2010).

Bone regeneration consists of cascades of complex biochemical processes that are coordinated via cells, ECM, and bioactive molecules such as osteogenic, angiogenic, inflammatory, and systemic GFs (Vo et al., 2012). The enhanced therapeutic effects of different GFs such as osteoinductive bone morphogenetic protein-2 (BMP-2) and mitogenic platelet-derived growth factor-BB (PDGF-BB) have been shown in clinical trials for bone defect treatments (Nevins et al., 2013; Shah et al., 2014). BMP-2 is an important member of TGF- $\beta$ superfamily that includes major modulators of osteogenesis, which play important roles in the commitment and differentiation of osteoprogenitors. BMP-2 loaded 2- $N, 6-O$-sulfated chitosan-based nanoparticles (S-NPs) were developed via complex coacervation of oppositely charged polyelectrolyte solutions as a delivery system for bone regeneration applications (Cao et al., 2014). In another study (Gan et al., 2015), pH-responsive chitosan-functionalized mesoporous silica nanoparticles were used for dual delivery of BMP-2 and dexamethasone (Dex) for osteoblast differentiation and bone regeneration at in vitro and in vivo conditions.

Neurotrophic factors (NF) including nerve growth factor (NGF), glialderived neurotrophic factor (GDNF), brain-derived neurotrophic factor (BDNF), insulin-like growth factor-1 (IGF-1), or basic FGF-2 are regulatory biological signals for promoting the development, survival, and regeneration of neurons (Harvey et al., 2014; Ziv-Polat et al., 2014). The alterations in 
cellular structure and metabolism of damaged neurons lead to impaired regeneration (Sivak et al., 2014). Biological factors can induce endogenous repair, enhance neural regeneration, and hinder inhibitor signals for the regeneration process (Donaghue et al., 2014). Delivery of these factors to the central nervous system (CNS) is a developing approach for treatments of traumatic brain, spinal cord, and peripheral nerve injuries and neural degenerative disorders (Mohtaram et al., 2013).

Different strategies have been developed for therapeutic delivery of biologics into the CNS such as liposomes, nanospheres, nanocapsules, dendrimers, and polymeric micelles (Orive et al., 2009). The design of nanosized delivery systems for the CNS focus on two important requirements: long circulation time of the nanocarrier systems consisting regenerating signals and ability to penetrate blood-brain barrier (BBB), which is formed by tight junctions preventing crossing of large neurotrophic protein molecules (Thorne and Frey II, 2001; Zhong and Bellamkonda, 2008). BDNF-bound magnetically guided nanoparticles overcame impermeability of BBB and decreased morphine-induced apoptosis in the CNS restoring the spine density and promoting regeneration of synaptic connections (PilakkaKanthikeel et al., 2013). In another study, bFGF-loaded PEG-PLGA nanoparticles were functionalized with Solanum tuberosum lectin (STL) for targeted delivery to brain tissue and enhanced spatial learning and memory of rats with Alzheimer's disease (AD) promoting the survival and neurite growth of neurons (Zhang et al., 2014).

The delivery systems loaded with multiple GFs can mimic complex in vivo conditions releasing different therapeutic biologics for effective tissue regeneration (Chen et al., 2010). Time-dependent controlled releases of multiple GFs at different stages of tissue regeneration via nanosized delivery systems enhance the biochemical processes sequentially. In one study (Perez et al., 2013), bone regeneration was induced by initial release of the angiogenic factors like VEGF or bFGF and then supported via BMP-2 release as an osteogenic factor.

Platelet-rich plasma consist of biologically active proteins including several GFs, such as the isoforms of platelet-derived growth factor (PDGF), transforming growth factor (TGF), and FGF (Intini, 2009). The lysates of plateletrich plasma are natural sources of multiple GFs that are important in bone regeneration (Visser et al., 2009). Hence, controlled delivery of plasma lysates via chitosan-chondroitin sulfate nanoparticles prepared by polyelectrolyte complexation was intriguing for bone tissue engineering applications promoting osteogenic differentiation via multiple factors (Santo et al., 2012).

Cytokines are small signaling molecules with sizes ranging between 8 and $40 \mathrm{kDa}$ and are responsible for regulating the immune response, 
inflammation, neoangiogenic processes, and cellular differentiation (Ioannidou, 2006). Cytokines are also important biochemical factors in tissue regeneration controlling cellular activity (Gelain et al., 2010). However, short half-lives and serum-mediated degradation of cytokines prevent therapeutic applications and lead to developing different strategies for delivery of these small biologics. Cytokine delivery to injury site by controlled delivery systems can direct tissue regeneration in the absence of the transplanted cells (Roche et al., 2013).

Inflammation process is one of the critical steps of regeneration, and this complex step is controlled by secretion of GFs and pro- or anti-inflammatory cytokines. Cytokine-mediated signaling of inflammation involving activation and proliferation of satellite cells is an essential component of muscle repair, regeneration, and growth (Tidball, 2005). Delivery of cytokines such as tumor necrosis factor- $\alpha$ (TNF- $\alpha$ ) (Chen et al., 2007), interferon- $\gamma$ (Cheng et al., 2008), or interleukin-6 (IL-6) (Serrano et al., 2008) mediating inflammation in muscle regeneration processes can be an alternative approach for the treatments of acute or chronic muscle damages.

\subsection{GENE AND SIRNA DELIVERY WITH NANOCARRIERS FOR TISSUE REGENERATION}

\subsubsection{Gene Delivery}

Gene therapy provides sustained expression of bioactive molecules including GFs and cytokines required for tissue regeneration (Bonadio et al., 1999) and can assist stem cells to differentiate into a variety of different lineages and cells types for regenerative medicine (Chen et al., 2011b). Low uptake across the cell membrane, limited stability of DNA molecules, and lack of nuclear targeting are the main difficulties in DNA delivery (Luo and Saltzman, 2000). Controlled delivery of DNA using nanosized carriers promotes gene delivery and extended transgene expression. In addition, targeted nanosized carriers for gene delivery can avoid side effects including immune response or distribution to the nontargeted tissue and cells (Pannier and Shea, 2004).

Delivery of DNA encoding for inductive biologics can spatially influence on cellular behavior and enhance the formation of complex architectures for tissue regeneration (De Laporte and Shea, 2007). Although viral and retroviral vectors have been showed to be highly efficient transfection agents for in vivo, nonviral systems provide lower immune response and controllable chemical and biological properties for gene delivery (Elangovan et al., 2014; 
Leong et al., 1998). Nonviral delivery nanocarriers for DNA are developed using a variety of synthetic and natural polymers such as PLL, PLG, PVA, PEG, poly(ethyleneimine) (PEI), collagen, hyaluronic acid, gelatin, or chitosan (Pannier and Shea, 2004).

Cationic lipids consisting of a positively charged head group, a hydrophobic chain, and a linker that joins the polar and nonpolar regions can interact with negatively charged DNA molecules and form complexes called "lipoplexes" (Bhattacharya and Bajaj, 2009; Gao and Hui, 2001; Tros de Ilarduya et al., 2010). Similar to cationic lipids, cationic polymers can also form complexes with DNA and form "polyplexes," which are capable of gene delivery into targeted cells (Zhang et al., 2004). PEI is a highly cationic polymer due to the presence of amino groups, and it can form nanoparticles by complexation with negatively charged plasmid DNA (pDNA) and prevents pDNA from lysosomal nuclease degradation (Pérez-Martínez et al., 2011). Chondrogenic differentiation of hMSCs was facilitated by the delivery of Sox 9 gene, an important transcription factor in the process of chondrogenesis, complexed with PEI on PLGA nanoparticles (Jeon et al., 2012). In another study, pDNA encoding Runt-related transcription factor 2 (RUNX2) was delivered in liposomes, which were immobilized at the surface of polycaprolactone (PCL) nanofiber meshes sustaining physical support for hMSCs during osteogenic differentiation (Monteiro et al., 2014). Bone regeneration in rat cranial defects was also enhanced via localized delivery of $\mathrm{PEI} / \mathrm{pDNA}$ nanocomplexes inducing expression of bone morphogenetic protein-4 (BMP-4) (Huang et al., 2005). On the other hand, cationic DNA/polymer nanocomplexes have some drawbacks including instabilities in physiological conditions and aggregations due to binding of serum proteins on the surfaces. Also cationic lipids and polymers (PEI) can be toxic. To eliminate these drawbacks, targeted delivery and internalization strategies have been developed for enhanced therapeutic activities.

Dendritic nanostructures are also promising gene delivery systems due to their highly branched structure, shape, and multivalency. These structures can be designed using biodegradable polymers providing nontoxicity and efficiency for gene delivery (Luo et al., 2014). Especially, polycationic dendrimers such as polyamidoamine (PAMAM) were shown to be particularly interesting in nucleic acid delivery providing high multivalent surface moiety, biocompatibility, and low cytotoxicity (Lee et al., 2014). Human bone morphogenetic protein-2 (hBMP-2) gene-containing PAMAM dendrimers were used as gene transfer carriers for inducing osteogenic differentiation of mesenchymal stem cells (MSCs) for bone regeneration applications (Santos et al., 2009). 


\subsection{2 siRNA Delivery}

Short interfering RNAs (siRNA) are a short double-stranded biomacromolecules that silence target gene expression as a consequence of binding to the corresponding messenger RNA. Gene therapy provides permanent genetic alteration, but only transient actions of protein therapeutics can be needed for tissue regeneration applications. Hence, siRNA-based strategies provide a safer alternative compared to the gene therapy. Delivery systems are required for siRNA due to siRNA's inability to diffuse easily to cell membrane related with its polyanionic nature, poor solubility, and readily degradation by enzymes (Yoo et al., 2011). Similar to DNA nanocarriers, different nonviral nanosized carriers have been developed for siRNAs using liposomes, lipoplexes, polymers, and also peptides (Gao and Huang, 2008).

Similar to pDNA/PEI complexation, siRNA can also interact with cationic polymers and form stable nonviral nanosized delivery systems. PEG-PEI/siRNA nanoparticles were used for suppressing the expression of Nogo receptor $(\mathrm{NgR})$, which has an important role in CNS controlling neural inflammation, arresting the neurite growths of injured neurons, and the differentiation of NSCs for CNS regeneration (Liang et al., 2012). Another siRNA delivery-based regeneration strategy was developed to increase the endogenous BMP levels through RNA silencing to downregulate BMP-2 antagonist NOGGIN glycoprotein using different nanosized carriers (Bassit et al., 2015).

\subsection{SYSTEMIC TARGETING AND CELLULAR INTERNALIZATION STRATEGIES FOR TISSUE REGENERATION}

\subsubsection{Targeted Delivery}

When small molecules or peptide/protein ligands bind to a receptor, they generally start a cascade of signaling events in cellular microenvironment. Systemic targeting of delivery systems can be sustained by ligand-receptor mediated or locally activated delivery via internal factors (specific enzymes or $\mathrm{pH}$ change) and external forces such as light or temperature (Bae and Park, 2011). There are specific ligands that can only bind to one type of receptor on a specific cell or tissue, and these specific ligands render the targeting of that specific tissue or cell type. The specificity also depends on the presence and expression of receptors on certain cells. In the natural process of regeneration, GFs bind to certain receptors on the cell surface and initiate a cellular response such as inflammation, recruitment of stem 
cells to the tissue, or angiogenesis. In addition, proteins take part in cell-to-cell signaling, which is crucial for a synchronized regeneration process. This receptor-protein interaction is so specific that only a certain protein or a protein family can recognize and bind to its receptor. Therefore, it is important to understand the natural regeneration process and have the knowledge of the expression patterns in each tissue at different regeneration stages to be able to engineer materials presenting specific ligands. These ligands will bind to certain target cells only, making it possible to induce a unique specific response of the body. As another advantage, the localization of the biologics in the target site will decrease the side effects of the treatment. One general concern about administration of biomacromolecules such as GFs is the induction of proliferation of cells and formation of benign tumors. This risk would be decreased via targeted delivery approaches, which can mimic the release of the natural GFs at therapeutic dosage and localization. Chemical ligands, antibodies, specific peptide sequences, and proteins are common strategies to target specific cells or microenvironments for sustained delivery of biomacromolecules via nanosized carriers.

2.4.1.1 Chemical Ligands There has been an intense effort to explore and synthesize chemical ligands that will target a specific tissue, and the knowledge of ECM composition in different tissues is invaluable for targeting. For example, bone tissue contains mineralized matrix in the form of hydroxyapatite, differently from other tissues, whereas cartilage contains high amounts of polysaccharides such as hyaluronic acid. Skin tissue contains elastin and fibrin for the formation of elastic fibers (Uitto et al., 1989). Using the knowledge about ECM composition, chemical molecules are synthesized to target different tissues. In one of the efforts, researchers synthesized a fullerene molecule that will target calcium phosphate mineral in hydroxyapatite of the bone. The synthesized molecule demonstrated high affinity to hydroxyapatite, and it was proposed as a potential chemical targeting ligand in bone regeneration (Gonzalez et al., 2002).

For cartilage targeting, derivatives of quaternary ammonium (QA) have been investigated in different studies. When the biodistribution of QAglucosamine (antirheumatic drug) conjugates was investigated, the conjugate of glucosamine demonstrated specificity for cartilage in a short time after injection (Giraud et al., 2000). Moreover, QA has been proposed to be effective for cartilage targeting of naproxen and ibuprofen in the treatment of arthritis (Pawar et al., 2012). Based on these studies, cartilagetargeting molecules might have an application in tissue engineering for the specific delivery of molecules. 
The proteins upregulated or exposed to cell surface after myocardial infarction (MI) were investigated for a potential in targeting the infarct area (Sy and Davis, 2010) for cardiac regeneration. p38 MAPK inhibitor, the chemical substance designed to bind p38, was delivered with polymeric microspheres to target $\mathrm{p} 38$ protein overexpressed after MI. The delivery system was shown to be effective in inhibiting cardiac dysfunction (Sy et al., 2008).

2.4.1.2 Antibodies Antibodies designed to bind a specific cell surface receptor hold great promise in the delivery of biologics for tissue regeneration. Antibody-targeted delivery is one of the focuses in drug delivery due to the specificity of antigen-antibody binding. There are many monoclonal antibody-based pharmaceutics in the market, but most of them were developed for cancer treatment (i.e., trastuzumab, bevacizumab, ibritumomab tiuxetan). On the other hand, their applications in tissue engineering are also expanding due to the several advantages of antibody targeting.

One of these approaches is the immobilization of an antibody to a scaffold for enhanced GF binding. With this strategy, not only cellspecific targeting but also spatiotemporal regulation of endogenous GFs can be mimicked. To mimic the spatial property, the local injection of the growth factor might be a basic strategy. On the other hand, a better strategy is to modulate the presence of the GF in the target tissue for a certain period of time. In a recent study, anti-BMP-2 monoclonal antibody was immobilized on different biomaterials for the purpose of capturing endogenous BMP-2 and inducing bone regeneration at the graft site (Ansari et al., 2014).

2.4.1.3 Peptides and Proteins Peptide ligands are also specific in terms of binding and might be more advantageous than protein ligands due to ease of synthesis and conjugation to the nanosized delivery systems. Peptide phage screening libraries provide the exploration of targeting peptides for a specific molecule and more specific purposes of use. Arginine-glycineaspartic acid (RGD) is one of the common ligands that have been used in tissue regeneration, which can increase the adhesion of cells by interacting with integrins on the cell surface.

Collagen-binding domain (CBD) is a promising peptide sequence for targeted delivery of nanosized carriers to collagen. GFs that are conjugated to $\mathrm{CBD}$ can be used to promote specific binding of GF to collagen. CBD-fused basic fibroblast growth factor (bFGF) was used for the regeneration of abdominal wall defects, and it was demonstrated to promote vascularization for 30 days after surgery (Shi et al., 2011). Similar to CBD, heparin-binding 
domain is used for targeted delivery purposes. The abundance of heparin in cartilage provides the targetability of cartilage with heparin-binding domain. In one study (Loffredo et al., 2014), IGF-1 was targeted to cartilage with heparin-binding domain and shown to be effective in osteoarthritis treatment in vivo.

Delivery of protein therapeutics such as NF to the CNS is highly limited due to BBB preventing diffusion of the macromolecules through the brain tissue. Cell-penetrating peptides (CPPs) can also be used as a brain-targeting strategy. 39-amino acid peptide derived from the rabies virus glycoprotein (RDP) containing specific nerve binding region was shown as an efficient protein carrier for targeting and delivering macromolecular proteins across the BBB (Fu et al., 2012).

Carbohydrate-binding proteins such as lectins are highly specific to monosugars or oligosaccharide structures (Wu et al., 2009). Lectinconjugated nanosized delivery systems can bind to glycosylated cell membrane components, and lectin-cell membrane interactions improve vesicular transport of the delivery systems enhancing penetration through biological barriers such as BBB, nasal mucosa, lung, buccal cavity, or eye (Bies et al., 2004). The surface modification of PEG-PLA nanoparticles with lectins enhanced the brain uptake after nasal administrations of the delivery system (Gao et al., 2007).

\subsubsection{Cellular Internalization Strategies}

Controlled delivery of biologics such as GFs, cytokines, nucleic acids, or siRNA is a promising approach in tissue engineering due to several advantages. However, the delivered biomacromolecules with nanosized carriers need to be internalized, and desired internal or external stimuli should be expressed from the target cell only after the internalization. A variety of internalization strategies have been developed since the internalization of biologics into cells is difficult due to electrostatic repulsion and the low uptake efficiencies of the nonviral carriers. The internalization strategies for nonviral carriers can be designed focusing on multiple uptake mechanisms including macropinocytosis, clathrin-mediated endocytosis, or caveolae-mediated endocytosis to promote transduction of the specific genes (Pérez-Martínez et al., 2011) required for the regeneration processes. Cellular internalization is also important for the delivery of the biologics into specific intracellular organelles such as the nucleus.

Peptide domains containing positively charged amino acids such as arginine and lysine have been reported to enhance uptake and cellular internalization of nanosized carriers including PAMAM dendrimers 
conjugated with L-arginine (Choi et al., 2004), arginine-chitosan/DNA self-assembled nanoparticles (Gao et al., 2008), arginine-grafted bioreducible poly(disulfide amine) polymers (ABP) for siRNA delivery (Beloor et al., 2012), oligoarginine-modified liposomes (Furuhata et al., 2006), and self-assembled oligoarginine-PEG-lipids for gene delivery (Maitani and Hattori, 2009). In addition, integration of CPP to the vectors or peptide nucleic acid conjugates can be used for internalization (Jung et al., 2011). Recently, CPP-decorated self-assembled peptide amphiphile (PA) nanospheres complexed with antisense oligonucleotides increased the internalization of the nanosized delivery system compared to nonbioactive PA nanostructures (Mumcuoglu et al., 2015).

Therapeutic proteins delivered with CPPs can be internalized in cells in the scope of tissue regeneration. Low molecular weight protamine was used as a CPP for the delivery of a transcription factor, transcriptional coactivator with PDZ-binding motif (TAZ). TAZ transcription factor was used for osteogenic differentiation of MSCs, and CPP-conjugated protein could translocate the cell membrane and conserved the functionality (Suh et al., 2014). In addition, production of induced pluripotent stem cells (iPSC) was shown to be possible with induction of CPP-conjugated proteins (Kim et al., 2009).

Cell-penetrating proteins also facilitate delivery of antisense oligonucleotides, which are designed for the modulation of expression levels of a specific gene. Antisense oligonucleotides and small interfering RNA (siRNA) have a great potential in tissue engineering since they can be engineered to target any gene. One antisense oligonucleotide was shown to accelerate the rate of wound healing by modulating expression of connexin 43 (Qiu et al., 2003). GFs can also be targeted by antisense oligonucleotides (Uchio et al., 2004), and the modulation of their expression might provide another way to control the presence of GFs during different stages of regeneration. CPP antisense oligonucleotide conjugates provide higher cellular internalization compared to naked oligonucleotides, and therefore, CPPs would render the antisense oligonucleotide-based therapy more successful. Antisense oligonucleotide designed to alter dystrophin transcript was delivered with arginine-rich CPPs, and the conjugate was systemically administered to restore muscle and cardiac dystrophin expression for promoting muscle and cardiac function (Yin et al., 2008). The peptides containing nuclear localization sequence (NLS) can be used as an alternative internalization strategy for nonviral nucleic acid delivery systems. Incorporation of the self-assembling SV40 peptide having T-antigen NLS with cationic liposomes for nuclear delivery promoted internalization and nuclear accumulation of the DNA (Rea et al., 2009). 


\subsection{FUTURE PERSPECTIVES}

Integration of nanotechnology, material science, and engineering has pioneered developments in advanced targeted and sustained nanosized delivery systems with enhanced therapeutic efficacies for tissue regeneration purposes. Targeted delivery, reduction in therapeutic dosage of biologics, controlled release, and multifunctionality are ultimate goals of developing nanosized delivery systems. The nanosized carriers for tissue regeneration provide significant health benefits including elimination of surgery or requirements of transplantations and low risks of immune responses.

Although different strategies and formulations have been examined for nanosized delivery systems in the preclinical studies, successful regeneration using external factors for different types of tissues in clinical applications is still challenging. There are different aspects that prevent the progress in clinical applicability of the designed nanosized delivery systems for tissue regeneration purposes. Manufacturing costs and problems with reproducible and scale-up production of advanced delivery systems prevent commercialization (Kearney and Mooney, 2013; Zhang et al., 2013) and clinical usage of the carriers for tissue engineering. Moreover, although chemical and physical properties of the nanosized delivery systems are characterized in detail, behavior of nanoscale carriers in in vivo conditions cannot be controlled effortlessly due to complex and dynamic nature of the cellular microenvironment (Ruenraroengsak et al., 2010). Successful regeneration can be induced via orchestrated neural, vascular, biochemical, and biophysical components of the tissues (Discher et al., 2009). On the other hand, the inadequate knowledge on repair processes and connections between overall signaling pathways delays the progress of sustained regeneration strategies using delivery systems (Rice et al., 2013).

To eliminate these drawbacks, different strategies have been studied to increase efficacy, stability, and targeting of the nanosized carriers. Since the release of single biologics is not sufficient to sustain continuous regeneration for complex tissues, the combined use of multiple factors synergistically provides a strategic advantage for overcoming the limitations (Borselli et al., 2010; Chen et al., 2010, 2011a; Hwang and Kloner, 2010; Shah et al., 2014). In addition, the delivery of multiple factors and developments of the multicomponent systems including delivering the cells and biologics together using suitable scaffolds promote therapeutic 
effectiveness and tissue regeneration capacity (Donaghue et al., 2014; Park et al., 2013).

Further improvement can be prolonged via incorporation of ECMmimetic scaffolds with nanosized delivery systems of biologics sustaining both biochemical and physical factors required for tissue repair and regeneration (Chung et al., 2007). Combination of the biological factorloaded nanocarriers with three-dimensional networks provides long-term controlled release of the bioactive molecules required for regeneration (Jeon et al., 2008). The incorporation of GF-loaded nanoparticles or nanosized delivery systems with three-dimensional scaffolds also enhances the efficiency of the released molecules for in vivo applications, preventing initial high burst release and enhancing bioactivity. For example, VEGF-encapsulated nanoparticles incorporated into threedimensional delivery systems such as PLGA scaffolds and Matrigel ${ }^{\mathrm{TM}}$ hydrogels enhanced angiogenesis in vivo (des Rieux et al., 2011). In another application, VEGF-loaded chitosan/heparin polyelectrolyte nanocomplexes immobilized into decellularized scaffolds promoted vascularization in mouse subcutaneous implantation model (Tan et al., 2011). Acidic FGF (aFGF)-loaded mesoporous silica nanospheres shelled by PLA were embedded into collagen scaffold, and the nanocarriers containing scaffold implanted in a rat subcutaneous tissue induced significant proliferation of osteoblast cells (Kim et al., 2014). In addition to the GFs, BMP-2 pDNA/chitosan nanoparticles were encapsulated into the fibrous network for slower release rate and enhanced biological activity (He et al., 2014; Nie and Wang, 2007). Furthermore, engineering of the bioactive molecules such as GFs with superaffinity to the scaffolds or carriers can be an alternative approach to prolonged delivery and increased efficacy and reducing the dosage required for the regenerative medicine applications (Martino et al., 2014).

Developments in fabrication techniques, understanding of tissue regeneration processes, and the roles of biological factors in detail will contribute to progress in this field. Combination of advantages of advanced nanosized delivery systems with the power of biological factors has also great potential to solve the recent problems in tissue regeneration applications. Industrial collaboration with scientific community can synergize the commercialization of the developed systems for therapeutic applications. Overall, it is believed that advanced nanosized delivery systems integrated with multicomponent designs will open new opportunities in delivery technologies and strategies for tissue regeneration. 


\section{REFERENCES}

Alvarez-Lorenzo, C. and Concheiro, A. (2014). Smart drug delivery systems: from fundamentals to the clinic. Chemical Communications 50, 7743-7765.

Alyautdin, R., Khalin, I., Nafeeza, M.I., Haron, M.H., and Kuznetsov, D. (2014). Nanoscale drug delivery systems and the blood-brain barrier. International Journal of Nanomedicine 9, 795.

Ansari, S., Freire, M.O., Pang, E.-K., Abdelhamid, A.I., Almohaimeed, M., and Zadeh, H.H. (2014). Immobilization of murine anti-BMP-2 monoclonal antibody on various biomaterials for bone tissue engineering. BioMed Research International 2014, 940860.

Bae, Y.H. and Park, K. (2011). Targeted drug delivery to tumors: myths, reality and possibility. Journal of Controlled Release 153, 198.

Bassit, A., Moffatt, P., Gaumond, M., and Hamdy, R. (2015). The potential use of nanoparticles for noggin siRNA delivery to accelerate bone formation in distraction osteogenesis. Journal of Nanomedicine and Nanotechnology 6, 2.

Beloor, J., Choi, C.S., Nam, H.Y., Park, M., Kim, S.H., Jackson, A., Lee, K.Y., Kim, S.W., Kumar, P., and Lee, S.-K. (2012). Arginine-engrafted biodegradable polymer for the systemic delivery of therapeutic siRNA. Biomaterials 33, 1640-1650.

Bhattacharya, S. and Bajaj, A. (2009). Advances in gene delivery through molecular design of cationic lipids. Chemical Communications, 4632-4656.

Bies, C., Lehr, C.-M., and Woodley, J.F. (2004). Lectin-mediated drug targeting: history and applications. Advanced Drug Delivery Reviews 56, 425-435.

Bonadio, J., Smiley, E., Patil, P., and Goldstein, S. (1999). Localized, direct plasmid gene delivery in vivo: prolonged therapy results in reproducible tissue regeneration. Nature Medicine 5, 753-759.

Borselli, C., Storrie, H., Benesch-Lee, F., Shvartsman, D., Cezar, C., Lichtman, J.W., Vandenburgh, H.H., and Mooney, D.J. (2010). Functional muscle regeneration with combined delivery of angiogenesis and myogenesis factors. Proceedings of the National Academy of Sciences 107, 3287-3292.

Cao, L., Wang, J., Hou, J., Xing, W., and Liu, C. (2014). Vascularization and bone regeneration in a critical sized defect using 2-N, 6-O-sulfated chitosan nanoparticles incorporating BMP-2. Biomaterials 35, 684-698.

Chen, S.-E., Jin, B., and Li, Y.-P. (2007). TNF- $\alpha$ regulates myogenesis and muscle regeneration by activating p38 MAPK. American Journal of Physiology-Cell Physiology 292, C1660-C1671.

Chen, F.-M., Zhang, M., and Wu, Z.-F. (2010). Toward delivery of multiple growth factors in tissue engineering. Biomaterials 31, 6279-6308.

Chen, F.-M., Wu, L.-A., Zhang, M., Zhang, R., and Sun, H.-H. (2011a). Homing of endogenous stem/progenitor cells for in situ tissue regeneration: promises, strategies, and translational perspectives. Biomaterials 32, 3189-3209. 
Chen, J., Chen, H., Li, P., Diao, H., Zhu, S., Dong, L., Wang, R., Guo, T., Zhao, J., and Zhang, J. (2011b). Simultaneous regeneration of articular cartilage and subchondral bone in vivo using MSCs induced by a spatially controlled gene delivery system in bilayered integrated scaffolds. Biomaterials 32, 4793-4805.

Cheng, M., Nguyen, M.-H., Fantuzzi, G., and Koh, T.J. (2008). Endogenous interferon- $\gamma$ is required for efficient skeletal muscle regeneration. American Journal of Physiology-Cell Physiology 294, C1183-C1191.

Choi, J.S., Nam, K., Park, J.-y., Kim, J.-B., Lee, J.-K., and Park, J.-s. (2004). Enhanced transfection efficiency of PAMAM dendrimer by surface modification with L-arginine. Journal of Controlled Release 99, 445-456.

Chung, Y.-I., Ahn, K.-M., Jeon, S.-H., Lee, S.-Y., Lee, J.-H., and Tae, G. (2007). Enhanced bone regeneration with BMP-2 loaded functional nanoparticlehydrogel complex. Journal of Controlled Release 121, 91-99.

Chung, Y.-I., Kim, S.-K., Lee, Y.-K., Park, S.-J., Cho, K.-O., Yuk, S.H., Tae, G., and Kim, Y.H. (2010). Efficient revascularization by VEGF administration via heparin-functionalized nanoparticle-fibrin complex. Journal of Controlled Release 143, 282-289.

Crafts, T.D., Jensen, A.R., Blocher-Smith, E.C., and Markel, T.A. (2015). Vascular endothelial growth factor: therapeutic possibilities and challenges for the treatment of ischemia. Cytokine 71, 385-393.

De Laporte, L. and Shea, L.D. (2007). Matrices and scaffolds for DNA delivery in tissue engineering. Advanced Drug Delivery Reviews 59, 292-307.

Discher, D.E., Mooney, D.J., and Zandstra, P.W. (2009). Growth factors, matrices, and forces combine and control stem cells. Science 324, 1673-1677.

Donaghue, I.E., Tam, R., Sefton, M.V., and Shoichet, M.S. (2014). Cell and biomolecule delivery for tissue repair and regeneration in the central nervous system. Journal of Controlled Release 190, 219-227.

Eichmann, A. and Simons, M. (2012). VEGF signaling inside vascular endothelial cells and beyond. Current Opinion in Cell Biology 24, 188-193.

Elangovan, S., D’Mello, S.R., Hong, L., Ross, R.D., Allamargot, C., Dawson, D.V., Stanford, C.M., Johnson, G.K., Sumner, D.R., and Salem, A.K. (2014). The enhancement of bone regeneration by gene activated matrix encoding for platelet derived growth factor. Biomaterials 35, 737-747.

Fleige, E., Quadir, M.A., and Haag, R. (2012). Stimuli-responsive polymeric nanocarriers for the controlled transport of active compounds: concepts and applications. Advanced Drug Delivery Reviews 64, 866-884.

Fu, A., Wang, Y., Zhan, L., and Zhou, R. (2012). Targeted delivery of proteins into the central nervous system mediated by rabies virus glycoprotein-derived peptide. Pharmaceutical Research 29, 1562-1569.

Furuhata, M., Kawakami, H., Toma, K., Hattori, Y., and Maitani, Y.(2006). Intracellular delivery of proteins in complexes with oligoarginine-modified liposomes and the effect of oligoarginine length. Bioconjugate Chemistry 17, 935-942. 
Gan, Q., Zhu, J., Yuan, Y., Liu, H., Qian, J., Li, Y., and Liu, C. (2015). A dual-delivery system of $\mathrm{pH}$-responsive chitosan-functionalized mesoporous silica nanoparticles bearing BMP-2 and dexamethasone for enhanced bone regeneration. Journal of Materials Chemistry B 3, 2056-2066.

Ganta, S., Devalapally, H., Shahiwala, A., and Amiji, M. (2008). A review of stimuli-responsive nanocarriers for drug and gene delivery. Journal of Controlled Release 126, 187-204.

Gao, K. and Huang, L. (2008). Nonviral methods for siRNA delivery. Molecular Pharmaceutics 6, 651-658.

Gao, H. and Hui, K. (2001). Synthesis of a novel series of cationic lipids that can act as efficient gene delivery vehicles through systematic heterocyclic substitution of cholesterol derivatives. Gene Therapy 8, 855-863.

Gao, X., Chen, J., Tao, W., Zhu, J., Zhang, Q., Chen, H., and Jiang, X. (2007). UEA I-bearing nanoparticles for brain delivery following intranasal administration. International Journal of Pharmaceutics 340, 207-215.

Gao, Y., Xu, Z., Chen, S., Gu, W., Chen, L., and Li, Y. (2008). Arginine-chitosan/ DNA self-assemble nanoparticles for gene delivery: in vitro characteristics and transfection efficiency. International Journal of Pharmaceutics 359, 241-246.

Gelain, F., Unsworth, L.D., and Zhang, S. (2010). Slow and sustained release of active cytokines from self-assembling peptide scaffolds. Journal of Controlled Release 145, 231-239.

Giraud, I., Rapp, M., Maurizis, J.-C., and Madelmont, J.-C. (2000). Application to a cartilage targeting strategy: synthesis and in vivo biodistribution of $14 \mathrm{C}$ labeled quaternary ammonium-glucosamine conjugates. Bioconjugate Chemistry 11, 212-218.

Golub, J.S., Kim, Y.-t., Duvall, C.L., Bellamkonda, R.V., Gupta, D., Lin, A.S., Weiss, D., Taylor, W.R., and Guldberg, R.E. (2010). Sustained VEGF delivery via PLGA nanoparticles promotes vascular growth. American Journal of Physiology-Heart and Circulatory Physiology 298, H1959-H1965.

Gonzalez, K.A., Wilson, L.J., Wu, W., and Nancollas, G.H. (2002). Synthesis and in vitro characterization of a tissue-selective fullerene: vectoring $\mathrm{C}_{60}(\mathrm{OH})_{16}$ AMBP to mineralized bone. Bioorganic \& Medicinal Chemistry 10, 1991-1997.

Gu, Z., Biswas, A., Zhao, M., and Tang, Y. (2011). Tailoring nanocarriers for intracellular protein delivery. Chemical Society Reviews 40, 3638-3655.

Harvey, A.R., Lovett, S.J., Majda, B.T., Yoon, J.H., Wheeler, L.P., and Hodgetts, S.I. (2014). Neurotrophic factors for spinal cord repair: which, where, how and when to apply, and for what period of time? Brain Research 1619, 36-71.

He, C., Nie, W., and Feng, W. (2014). Engineering of biomimetic nanofibrous matrices for drug delivery and tissue engineering. Journal of Materials Chemistry B 2, 7828-7848. 
Heo, D.N., Ko, W.-K., Bae, M.S., Lee, J.B., Lee, D.-W., Byun, W., Lee, C.H., Kim, E.-C., Jung, B.-Y., and Kwon, I.K. (2014). Enhanced bone regeneration with a gold nanoparticle-hydrogel complex. Journal of Materials Chemistry B 2, 1584-1593.

Howard, D., Buttery, L.D., Shakesheff, K.M., and Roberts, S.J. (2008). Tissue engineering: strategies, stem cells and scaffolds. Journal of Anatomy 213, 66-72.

Huang, Y., Simmons, C., Kaigler, D., Rice, K., and Mooney, D. (2005). Bone regeneration in a rat cranial defect with delivery of PEI-condensed plasmid DNA encoding for bone morphogenetic protein-4 (BMP-4). Gene Therapy 12, 418-426.

Huang, M., Vitharana, S.N., Peek, L.J., Coop, T., and Berkland, C. (2007). Polyelectrolyte complexes stabilize and controllably release vascular endothelial growth factor. Biomacromolecules 8, 1607-1614.

Hwang, H. and Kloner, R.A. (2010). Improving regenerating potential of the heart after myocardial infarction: factor-based approach. Life Sciences 86, $461-472$.

Intini, G. (2009). The use of platelet-rich plasma in bone reconstruction therapy. Biomaterials 30, 4956-4966.

Ioannidou, E. (2006). Therapeutic modulation of growth factors and cytokines in regenerative medicine. Current Pharmaceutical Design 12, 2397-2408.

Jeon, O., Song, S.J., Yang, H.S., Bhang, S.-H., Kang, S.-W., Sung, M.A., Lee, J.H., and Kim, B.-S. (2008). Long-term delivery enhances in vivo osteogenic efficacy of bone morphogenetic protein-2 compared to short-term delivery. Biochemical and Biophysical Research Communications 369, 774-780.

Jeon, S.Y., Park, J.S., Yang, H.N., Woo, D.G., and Park, K.-H. (2012). Co-delivery of SOX9 genes and anti-Cbfa-1 siRNA coated onto PLGA nanoparticles for chondrogenesis of human MSCs. Biomaterials 33, 4413-4423.

Jung, M.-R., Shim, I.-K., Kim, E.-S., Park, Y.-J., Yang, Y.-I., Lee, S.-K., and Lee, S.-J. (2011). Controlled release of cell-permeable gene complex from poly (L-lactide) scaffold for enhanced stem cell tissue engineering. Journal of Controlled Release 152, 294-302.

Kearney, C.J. and Mooney, D.J. (2013). Macroscale delivery systems for molecular and cellular payloads. Nature Materials 12, 1004-1017.

Khandare, J., Calderón, M., Dagia, N.M., and Haag, R. (2012). Multifunctional dendritic polymers in nanomedicine: opportunities and challenges. Chemical Society Reviews 41, 2824-2848.

Kim, D., Kim, C.-H., Moon, J.-I., Chung, Y.-G., Chang, M.-Y., Han, B.-S., Ko, S., Yang, E., Cha, K.Y., and Lanza, R. (2009). Generation of human induced pluripotent stem cells by direct delivery of reprogramming proteins. Cell Stem Cell 4, 472. 
Kim, T.-H., Eltohamy, M., Kim, M., Perez, R.A., Kim, J.-H., Yun, Y.-R., Jang, J.-H., Lee, E.-J., Knowles, J.C., and Kim, H.-W. (2014). Therapeutic foam scaffolds incorporating biopolymer-shelled mesoporous nanospheres with growth factors. Acta Biomaterialia 10, 2612-2621.

Ko, W.-K., Heo, D.N., Moon, H.-J., Lee, S.J., Bae, M.S., Lee, J.B., Sun, I.-C., Jeon, H.B., Park, H.K., and Kwon, I.K. (2015). The effect of gold nanoparticle size on osteogenic differentiation of adipose-derived stem cells. Journal of Colloid and Interface Science 438, 68-76.

Kumari, A., Yadav, S.K., and Yadav, S.C. (2010). Biodegradable polymeric nanoparticles based drug delivery systems. Colloids and Surfaces B: Biointerfaces $75,1-18$.

Laurencin, C.T., Ashe, K.M., Henry, N., Kan, H.M., and Lo, K.W.-H. (2014). Delivery of small molecules for bone regenerative engineering: preclinical studies and potential clinical applications. Drug Discovery Today 19, 794-800.

Lee, J., Jung, J., Kim, Y.-J., Lee, E., and Choi, J.S. (2014). Gene delivery of PAMAM dendrimer conjugated with the nuclear localization signal peptide originated from fibroblast growth factor 3. International Journal of Pharmaceutics 459, 10-18.

Leong, K., Mao, H.-Q., Truong-Le, V., Roy, K., Walsh, S., and August, J. (1998). DNA-polycation nanospheres as non-viral gene delivery vehicles. Journal of Controlled Release 53, 183-193.

Liang, Y., Liu, Z., Shuai, X., Wang, W., Liu, J., Bi, W., Wang, C., Jing, X., Liu, Y., and Tao, E. (2012). Delivery of cationic polymer-siRNA nanoparticles for gene therapies in neural regeneration. Biochemical and Biophysical Research Communications 421, 690-695.

Loffredo, F.S., Pancoast, J.R., Cai, L., Vannelli, T., Dong, J.Z., Lee, R.T., and Patwari, P. (2014). Targeted delivery to cartilage is critical for in vivo efficacy of insulin-like growth factor 1 in a rat model of osteoarthritis. Arthritis \& Rheumatology 66, 1247-1255.

Luo, D. and Saltzman, W.M. (2000). Synthetic DNA delivery systems. Nature Biotechnology 18, 33-37.

Luo, K., He, B., Wu, Y., Shen, Y., and Gu, Z. (2014). Functional and biodegradable dendritic macromolecules with controlled architectures as nontoxic and efficient nanoscale gene vectors. Biotechnology Advances 32, 818-830.

Maitani, Y. and Hattori, Y. (2009). Oligoarginine-PEG-lipid particles for gene delivery. Expert Opinion on Drug Delivery 6, 1065-1077.

Martino, M.M., Briquez, P.S., Güç, E., Tortelli, F., Kilarski, W.W., Metzger, S., Rice, J.J., Kuhn, G.A., Müller, R., and Swartz, M.A. (2014). Growth factors engineered for super-affinity to the extracellular matrix enhance tissue healing. Science $343,885-888$. 
McCall, J.D., Lin, C.-C., and Anseth, K.S. (2011). Affinity peptides protect transforming growth factor beta during encapsulation in poly (ethylene glycol) hydrogels. Biomacromolecules 12, 1051-1057.

Mishra, B., Patel, B.B., and Tiwari, S. (2010). Colloidal nanocarriers: a review on formulation technology, types and applications toward targeted drug delivery. Nanomedicine: Nanotechnology, Biology and Medicine 6, 9-24.

Mohtaram, N.K., Montgomery, A., and Willerth, S.M. (2013). Biomaterial-based drug delivery systems for the controlled release of neurotrophic factors. Biomedical Materials 8, 022001.

Monteiro, N., Ribeiro, D., Martins, A., Faria, S., Fonseca, N.A., Moreira, J.N., Reis, R.L., and Neves, N.M. (2014). Instructive nanofibrous scaffold comprising runt-related transcription factor 2 gene delivery for bone tissue engineering. ACS Nano 8, 8082-8094.

Mumcuoglu, D., Sardan, M., Tekinay, T., Guler, M.O., and Tekinay, A.B. (2015). Oligonucleotide delivery with cell surface binding and cell penetrating peptide amphiphile nanospheres. Molecular Pharmaceutics 12, 1584-1591.

Nevins, M., Kao, R.T., McGuire, M.K., McClain, P.K., Hinrichs, J.E., McAllister, B.S., Reddy, M.S., Nevins, M.L., Genco, R.J., and Lynch, S.E. (2013). Plateletderived growth factor promotes periodontal regeneration in localized osseous defects: 36-month extension results from a randomized, controlled, doublemasked clinical trial. Journal of Periodontology 84, 456-464.

Nie, H. and Wang, C.-H. (2007). Fabrication and characterization of PLGA/HAp composite scaffolds for delivery of BMP-2 plasmid DNA. Journal of Controlled Release 120, 111-121.

Novoa-Carballal, R., Silva, C., Möller, S., Schnabelrauch, M., Reis, R.L., and Pashkuleva, I. (2014). Tunable nano-carriers from clicked glycosaminoglycan block copolymers. Journal of Materials Chemistry B 2, 4177-4184.

Orive, G., Anitua, E., Pedraz, J.L., and Emerich, D.F. (2009). Biomaterials for promoting brain protection, repair and regeneration. Nature Reviews Neuroscience 10, 682-692.

Pannier, A.K. and Shea, L.D. (2004). Controlled release systems for DNA delivery. Molecular Therapy 10, 19-26.

Panyam, J. and Labhasetwar, V. (2003). Biodegradable nanoparticles for drug and gene delivery to cells and tissue. Advanced Drug Delivery Reviews 55, 329-347.

Park, S.-Y., Kim, K.-H., Shin, S.-Y., Koo, K.-T., Lee, Y.-M., and Seol, Y.-J. (2013). Dual delivery of rhPDGF-BB and bone marrow mesenchymal stromal cells expressing the BMP2 gene enhance bone formation in a critical-sized defect model. Tissue Engineering Part A 19, 2495-2505.

Pawar, V., Gohil, V., Tamboli, R., Giridhar, R., and Yadav, M.R. (2012). Cartilage targeted chemical delivery of naproxen and ibuprofen for the treatment of arthritis. Journal of Pharmacy \& Bioallied Sciences 4, S10. 
Perez, R.A., Won, J.-E., Knowles, J.C., and Kim, H.-W. (2013). Naturally and synthetic smart composite biomaterials for tissue regeneration. Advanced Drug Delivery Reviews 65, 471-496.

Pérez-Martínez, F.C., Guerra, J., Posadas, I., and Ceña, V. (2011). Barriers to nonviral vector-mediated gene delivery in the nervous system. Pharmaceutical Research 28, 1843-1858.

Pilakka-Kanthikeel, S., Atluri, V.S.R., Sagar, V., Saxena, S.K., and Nair, M. (2013). Targeted brain derived neurotrophic factors (BDNF) delivery across the blood-brain barrier for neuro-protection using magnetic nano carriers: an in-vitro study. PLoS One 8, e62241.

Place, L.W., Sekyi, M., and Kipper, M.J. (2014). Aggrecan-mimetic, glycosaminoglycan-containing nanoparticles for growth factor stabilization and delivery. Biomacromolecules 15, 680-689.

Ponte, A.L., Marais, E., Gallay, N., Langonne, A., Delorme, B., Herault, O., Charbord, P., and Domenech, J. (2007). The in vitro migration capacity of human bone marrow mesenchymal stem cells: comparison of chemokine and growth factor chemotactic activities. Stem Cells 25, 1737-1745.

Qiu, C., Coutinho, P., Frank, S., Franke, S., Law, L.-y., Martin, P., Green, C.R., and Becker, D.L. (2003). Targeting connexin43 expression accelerates the rate of wound repair. Current Biology 13, 1697-1703.

Rea, J.C., Gibly, R.F., Barron, A.E., and Shea, L.D. (2009). Self-assembling peptide-lipoplexes for substrate-mediated gene delivery. Acta Biomaterialia 5, 903-912.

Rice, J.J., Martino, M.M., De Laporte, L., Tortelli, F., Briquez, P.S., and Hubbell, J.A. (2013). Engineering the regenerative microenvironment with biomaterials. Advanced Healthcare Materials 2, 57-71.

des Rieux, A., Ucakar, B., Mupendwa, B.P.K., Colau, D., Feron, O., Carmeliet, P., and Préat, V. (2011). 3D systems delivering VEGF to promote angiogenesis for tissue engineering. Journal of Controlled Release 150, 272-278.

Roche, S., D’Ippolito, G., Gomez, L.A., Bouckenooghe, T., Lehmann, S., Montero-Menei, C.N., and Schiller, P.C. (2013). Comparative analysis of protein expression of three stem cell populations: models of cytokine delivery system in vivo. International Journal of Pharmaceutics 440, 72-82.

Ruenraroengsak, P., Cook, J.M., and Florence, A.T. (2010). Nanosystem drug targeting: facing up to complex realities. Journal of Controlled Release 141, 265-276.

Santo, V.E., Gomes, M.E., Mano, J.F., and Reis, R.L. (2012). Chitosan-chondroitin sulphate nanoparticles for controlled delivery of platelet lysates in bone regenerative medicine. Journal of Tissue Engineering and Regenerative Medicine 6, s47-s59.

Santos, J.L., Oramas, E., Pêgo, A.P., Granja, P.L., and Tomás, H. (2009). Osteogenic differentiation of mesenchymal stem cells using PAMAM dendrimers as gene delivery vectors. Journal of Controlled Release 134, 141-148. 
Serrano, A.L., Baeza-Raja, B., Perdiguero, E., Jardí, M., and Muñoz-Cánoves, P. (2008). Interleukin-6 is an essential regulator of satellite cell-mediated skeletal muscle hypertrophy. Cell Metabolism 7, 33-44.

Shah, N.J., Hyder, M.N., Quadir, M.A., Courchesne, N.-M.D., Seeherman, H.J., Nevins, M., Spector, M., and Hammond, P.T. (2014). Adaptive growth factor delivery from a polyelectrolyte coating promotes synergistic bone tissue repair and reconstruction. Proceedings of the National Academy of Sciences 111, 12847-12852.

Shi, C., Chen, W., Zhao, Y., Chen, B., Xiao, Z., Wei, Z., Hou, X., Tang, J., Wang, Z., and Dai, J. (2011). Regeneration of full-thickness abdominal wall defects in rats using collagen scaffolds loaded with collagen-binding basic fibroblast growth factor. Biomaterials 32, 753-759.

Sivak, W.N., White, J.D., Bliley, J.M., Tien, L.W., Liao, H.T., Kaplan, D.L., and Marra, K.G. (2014). Delivery of chondroitinase ABC and glial cell line-derived neurotrophic factor from silk fibroin conduits enhances peripheral nerve regeneration. Journal of Tissue Engineering and Regenerative Medicine. doi:10.1002/term.1970

Suh, J.S., Lee, J.Y., Choi, Y.J., You, H.K., Hong, S.-D., Chung, C.P., and Park, Y.J. (2014). Intracellular delivery of cell-penetrating peptide-transcriptional factor fusion protein and its role in selective osteogenesis. International Journal of Nanomedicine 9, 1153.

Sy, J.C. and Davis, M.E. (2010). Delivering regenerative cues to the heart: cardiac drug delivery by microspheres and peptide nanofibers. Journal of Cardiovascular Translational Research 3, 461-468.

Sy, J.C., Seshadri, G., Yang, S.C., Brown, M., Oh, T., Dikalov, S., Murthy, N., and Davis, M.E. (2008). Sustained release of a 338 inhibitor from non-inflammatory microspheres inhibits cardiac dysfunction. Nature Materials 7, 863-868.

Tan, Q., Tang, H., Hu, J., Hu, Y., Zhou, X., Tao, Y., and Wu, Z. (2011). Controlled release of chitosan/heparin nanoparticle-delivered VEGF enhances regeneration of decellularized tissue-engineered scaffolds. International Journal of Nanomedicine 6, 929-942.

Tessmar, J.K. and Göpferich, A.M. (2007). Matrices and scaffolds for protein delivery in tissue engineering. Advanced Drug Delivery Reviews 59, 274-291.

Thorne, R.G. and Frey II, W.H. (2001). Delivery of neurotrophic factors to the central nervous system. Clinical Pharmacokinetics 40, 907-946.

Tidball, J.G. (2005). Inflammatory processes in muscle injury and repair. American Journal of Physiology-Regulatory, Integrative and Comparative Physiology 288, R345-R353.

Tros de Ilarduya, C., Sun, Y., and Düzgüneş, N. (2010). Gene delivery by lipoplexes and polyplexes. European Journal of Pharmaceutical Sciences 40, 159-170. 
Uchio, K., Graham, M., Dean, N.M., Rosenbaum, J., and Desmoulière, A. (2004). Down-regulation of connective tissue growth factor and type I collagen mRNA expression by connective tissue growth factor antisense oligonucleotide during experimental liver fibrosis. Wound Repair and Regeneration 12, 60-66.

Uitto, J., Olsen, D.R., and Fazio, M.J. (1989). Extracellular matrix of the skin: 50 years of progress. Journal of Investigative Dermatology 92, 61S-77S.

Vermonden, T., Censi, R., and Hennink, W.E. (2012). Hydrogels for protein delivery. Chemical Reviews 112, 2853-2888.

Visser, L.C., Arnoczky, S.P., Caballero, O., Kern, A., Ratcliffe, A., and Gardner, K.L. (2009). Growth factor-rich plasma increases tendon cell proliferation and matrix synthesis on a synthetic scaffold: an in vitro study. Tissue Engineering Part A 16, 1021-1029.

Vo, T.N., Kasper, F.K., and Mikos, A.G. (2012). Strategies for controlled delivery of growth factors and cells for bone regeneration. Advanced Drug Delivery Reviews 64, 1292-1309.

Wang, N.X. and von Recum, H.A. (2011). Affinity-based drug delivery. Macromolecular Bioscience 11, 321-332.

Wei, G. and Ma, P.X. (2008). Nanostructured biomaterials for regeneration. Advanced Functional Materials 18, 3568-3582.

Wu, A.M., Lisowska, E., Duk, M., and Yang, Z. (2009). Lectins as tools in glycoconjugate research. Glycoconjugate Journal 26, 899-913.

Yin, H., Moulton, H.M., Seow, Y., Boyd, C., Boutilier, J., Iverson, P., and Wood, M.J. (2008). Cell-penetrating peptide-conjugated antisense oligonucleotides restore systemic muscle and cardiac dystrophin expression and function. Human Molecular Genetics 17, 3909-3918.

Yoo, J.-W., Irvine, D.J., Discher, D.E., and Mitragotri, S. (2011). Bio-inspired, bioengineered and biomimetic drug delivery carriers. Nature Reviews Drug Discovery 10, 521-535.

Young, S., Wong, M., Tabata, Y., and Mikos, A.G. (2005). Gelatin as a delivery vehicle for the controlled release of bioactive molecules. Journal of Controlled Release 109, 256-274.

Zhang, S., Xu, Y., Wang, B., Qiao, W., Liu, D., and Li, Z. (2004). Cationic compounds used in lipoplexes and polyplexes for gene delivery. Journal of Controlled Release 100, 165-180.

Zhang, Y., Chan, H.F., and Leong, K.W. (2013). Advanced materials and processing for drug delivery: the past and the future. Advanced Drug Delivery Reviews 65, 104-120.

Zhang, C., Chen, J., Feng, C., Shao, X., Liu, Q., Zhang, Q., Pang, Z., and Jiang, X. (2014). Intranasal nanoparticles of basic fibroblast growth factor for brain delivery to treat Alzheimer's disease. International Journal of Pharmaceutics $461,192-202$. 
Zhong, Y. and Bellamkonda, R.V. (2008). Biomaterials for the central nervous system. Journal of the Royal Society Interface 5, 957-975.

Ziv-Polat, O., Shahar, A., Levy, I., Skaat, H., Neuman, S., Fregnan, F., Geuna, S., Grothe, C., Haastert-Talini, K., and Margel, S. (2014). The role of neurotrophic factors conjugated to iron oxide nanoparticles in peripheral nerve regeneration: in vitro studies. BioMed Research International 2014, 10. 